\title{
Special issue on "Towards the connected body: advances in body communications"
}

\author{
Philippe De Doncker ${ }^{1 *}$, Jun-Ichi Takada ${ }^{2}$, Arie Reichman $^{3}$ and Dries Neirynck ${ }^{4}$
}

Body area networks (BANs) are quickly becoming a key technology for wireless communications. They consist of body-worn or implanted sensors that wirelessly communicate, leading either to in- or on-body communications between sensors, or to off-body communications with devices located in the close vicinity. BAN's potential applications include, among others, $m$-health information systems collecting vital parameters in real-time, high-speed connections over the body surface for multimedia transfers or human body interactions with its environment for augmented reality devices.

Several technologies are currently under development in order to enable these communications, either narrowband (including RFID technologies), or ultra-wideband (IR-UWB). The latter could be advantageously coupled with positioning algorithms for simultaneous body motion capture. However, in practice, numerous challenges still need to be tackled in order to make BANs viable. The sensor's autonomy, size, and cost are critical parameters which can only be optimized by adopting an interdisciplinary approach, where the propagation phenomena, the coding or relaying strategies, the MAC protocols, and the network topology are strongly interrelated. The coexistence issue with other networks also seems to be crucial, especially in the hospital environment.

This special issue goes through all the layers of telecommunication systems dedicated to body communications, from channel models for on- or off-body communications, to global telecare systems based on Bluetooth, smartphones, and GPS.

The article "Experimental characterization of an UWB channel for body area networks" by P. Chiang et al. presents UWB channel measurement campaigns, for both on- and off-body communications. The measured path loss and multipath analysis suggest that a LOS UWB channel is excellent for low-power, high-data-rate

\footnotetext{
* Correspondence: pdedonck@ulb.ac.be

'Université libre de Bruxelles, Bruxelles, Belgium

Full list of author information is available at the end of the article
}

transmission, while NLOS and on-body channels need to be reconfigured to operate at a lower data rate due to high path loss.

The article "An analytical modeling of polarized timevariant on-body propagation channels with dynamic body scattering" by L. Liu et al. presents an analytical model of the on-body scattering and propagation on the trunk of a walking person. The results act as an example of how analytical models can provide efficient yet precise predictions of time-variant, on-body propagation to understand the mechanisms underlying the propagation characteristics.

The article "In-body Path Loss Models for Implants in Heterogeneous Human Tissues using Implantable Slot Dipole Conformal Flexible Antennas" by F. Axisa et al. discusses the path loss of implant communication channel for a 6-year male child with slot dipole conformal antennas at $2.45 \mathrm{GHz}$ via 3D electromagnetic simulations. The article proves that exponential attenuation model is well applicable to the heterogeneous model as well, but homogeneous phantom may underestimate the pathloss compared with more realistic heterogeneous phantom.

The article "Secure Precise Clock Synchronization for Interconnected Body Area Networks" by D. Sanchez et al. tackles secure time synchronization in BANs. A combination of secure pairwise synchronization, rate adaptive time synchronization, and $\mu$ TESLA is used to achieve energy efficient, accurate, and secure synchronization between BAN nodes.

In "Emergency Handling for MAC protocol in Human Body Communication (HBC-MAC)" by O. Buyanjargal et al., a specific emergency handling operation is proposed for BAN's MAC protocol in order to meet the emergency requirements for BAN without wasteful dedicated slots for emergency in each superframe.

The article "Impact of the Environment and the Topology on the Performance of Hierarchical Body Area Networks" by J.-M. Dricot et al. addresses the throughput and energy consumption of various BAN topologies 
in both indoor and outdoor environments. The presented framework helps system architects to select the most suitable MAC scheme for their application.

The article "Quality of Service (QoS) Regulation in Secure Body Area Networks: System Modeling and Adaptation Methods" by Fr. Minhthang Bui, and D. Hatzinakos, studies signal processing methods for delivering secure communications in BAN, particularly when using biometrics. An optimization framework is presented to aggregate various methods, enabling overall quality of service (QoS) regulation in an integrated and flexible manner and effective in managing security solutions for BAN.

The article "Titan: An Enabling Framework for Activity-Aware PervasiveApps in Opportunistic Personal Area Networks" by D. Roggen et al. introduces Titan: a service-oriented framework to support the design, development, deployment, and execution of activity-aware pervasive computing applications that use available sensors for activity recognition in open-ended environments where resource availability cannot be controlled and predicted.

Finally, the article "Development and Evaluation of a Python Telecare System based on a Bluetooth Body Area Network" by M. J. Morón et al. presents a telemonitoring system, based on Bluetooth, GPS, and a smartphone that forwards the data received from the Bluetooth devices to a central server that provides universal access to the information of the patient's location and health status through a web application.

\section{Author details}

${ }^{1}$ Université libre de Bruxelles, Bruxelles, Belgium ${ }^{2}$ Tokyo Institute of Technology, Tokyo, Japan ${ }^{3}$ Ruppin Academic Center, Tel Aviv University, Tel Aviv, Israel ${ }^{4}$ DecaWave, Dublin, Ireland

\section{Competing interests}

The authors declare that they have no competing interests.

Received: 25 August 2011 Accepted: 16 December 2011 Published: 16 December 2011 doi:10.1186/1687-1499-2011-203

Cite this article as: De Doncker et al:: Special issue on "Towards the connected body: advances in body communications". EURASIP Journal on Wireless Communications and Networking 2011 2011:203.

\section{Submit your manuscript to a SpringerOpen ${ }^{\mathcal{O}}$ journal and benefit from:}

- Convenient online submission

- Rigorous peer review

- Immediate publication on acceptance

- Open access: articles freely available online

- High visibility within the field

- Retaining the copyright to your article

Submit your next manuscript at $\gg$ springeropen.com 